\title{
Towards Automated Electron Microscopy Image Segmentation for Nanoparticles of Complex Shape by Convolutional Neural Networks
}

\author{
Bastian Rühle and Vasile-Dan Hodoroaba
}

\section{Federal Institute for Materials Research and Testing (BAM), Berlin, Berlin, Germany}

The reliable physico-chemical characterization of nanoparticles (NPs) begins with the accurate measurement of NP size (distributions) and continues with the determination of other relevant parameters such as shape, structure, chemical composition, specific surface area, surface chemistry, etc. Particularly when the shape of the NPs deviates significantly from spherical, even a 'simple' measurement such as nanoparticle size constitutes a challenging analytical task for all available sizing methods, including various forms of electron microscopy [1]. Furthermore, the degree of NP agglomeration/aggregation alters the final result of the particle size distribution considerably [1], and a sample preparation yielding isolated, contamination-free NPs that are dispersed homogeneously on a suitable substrate requires well-developed protocols which are often not available for industrial NPs [1,2]. The capability of electron microscopy to assess the size and shape of individual NPs and directly yield a number-weighted size distribution (as opposed to a volume or intensity weighted distribution) is counterbalanced by the reduced number of analyzed NPs in comparison to ensemble sizing techniques such as DLS, PTA, SAXS etc.. On top, there is a great need for automated acquisition of electron micrographs and automated image analysis rather than a tedious manual measurement of individual particles that is prone to errors and operator bias in order to get representative particle size distributions for a statistically relevant number of NPs.

For 'ideal' NPs with simple morphology, i.e. (nearly) spherical and non-agglomerated, such as silica or gold NPs, the automated measurement of the size distribution works accurately [3]. For more complex NPs, the automated image analysis can be resolved by machine learning algorithms starting with manual identification (segmentation) of a reduced number of NPs (including thresholding based on physical modelling).

Neural networks, especially Convolutional Neural Networks (CNNs) have shown enormous potential in image classification and segmentation tasks, and their scope has been extended towards automated image analysis of biomedical and life science data such as light microscopy images or CT/MRI tomography [5-7]. However, there is a severe lack of annotated, high quality image data for training, validating, and comparing different algorithms for the segmentation of NPs in electron microscopy images. In some cases, such training data can be generated semi-automatically from e.g. TEM images followed by a manual correction of the resulting segmentation masks, but in other cases this approach fails as well. Hence, we explored different ways of generating suitable training data for 'non-ideal' samples using various approaches, e.g., computer-generated images or unsupervised learning algorithms such as generative adversarial networks (GANs). We used these data to train simple CNNs to produce segmentation masks of SEM images and tested the trained networks on real SEM images of complex nanoparticle samples. Figure 1 shows an example of the segmentation and measurement of particle size distribution of a complex, agglomerated $\mathrm{BaSO}_{4} \mathrm{NP}$ sample from an SEM micrograph by manual evaluation (ImageJ) and by using automated particle segmentation with convolutional neural networks $(\mathrm{CNN})$.

In conclusion, we can say that the novel use of CNN for the automated analysis of the size of NPs of complex shape and with a high degree of agglomeration has proved to be a promising tool for the evaluation of particle size distribution on a large number of constituent particles. Further development and validation of the preliminary model, respectively larger training and validation data sets are necessary [8]. 
a) Manual
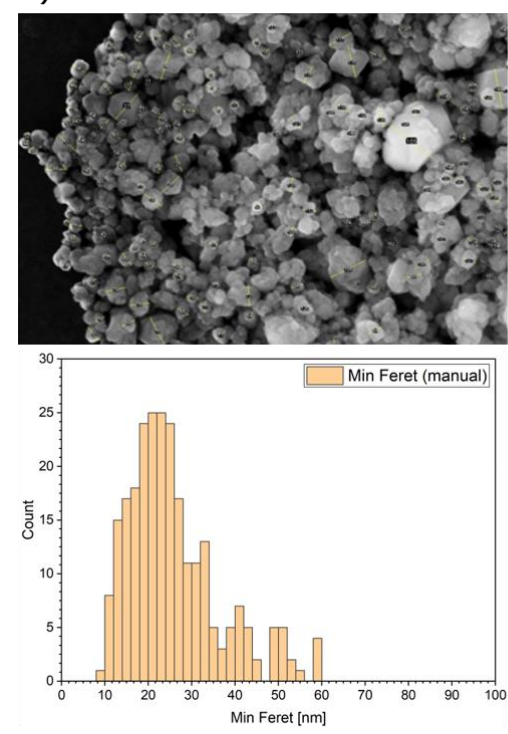

\begin{tabular}{ll}
\hline & Manual \\
\hline $\mathrm{N}$ & 265 \\
\hline Mean /nm & 28 \\
Std. dev. /nm & 16 \\
\hline Median /nm & 24 \\
\hline
\end{tabular}

b) CNN (UNet)
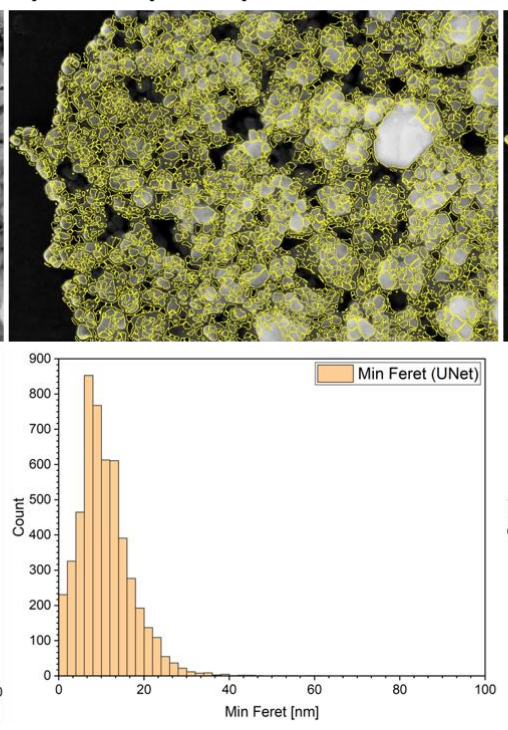

CNN (UNet)

5137

7

10 c) CNN (MultiResUNet)
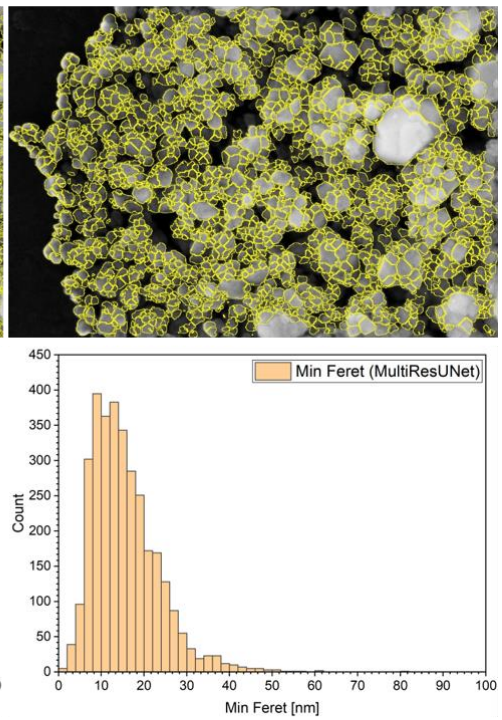

CNN (MultiResUNet)

3223

16

8

14

Figure 1. Particle size measurement of agglomerated BaSO4 nanoparticles in an SEM image by a) manual analysis of the smallest dimension (minimum Feret), b) by image segmentation via as UNet-CNN, and c) by image segmentation via a MultiResUNet-CNN [7]. The resulting particle size distributions (number of analyzed nanoparticles, mean and its standard deviation and median values) are given in the table below

\section{References}

[1] F Babick et al, J. Nanopart. Res. 18:158 (2016), pp. 1-40.

[2] U Mansfeld et al, Microsc. Microanal. 25 (2019), p. 2328.

[3] P-J De Temmerman et al, J. Nanopart. Res. (2014), 16:2177.

[4] Uusimaeki et al, J. Nanopart. Res. 21:122 (2019), pp. 1-11.

[5] O Renneberg et al., arXiv:1505.04597.

[6] L Lu et al., IEEE Transactions on Medical Imaging 35:5 (2016), pp. 1170 - 1181.

[7] N Ibtehaz et al., arXiv: 1902.04049v1.

[8] This project has received funding from the EMPIR programme co-financed by the Participating States and from the European Union's Horizon 2020 research and innovation Programme. 